Article

\title{
Systemic Effects of mitoTEMPO upon Lipopolysaccharide Challenge Are Due to Its Antioxidant Part, While Local Effects in the Lung Are Due to Triphenylphosphonium
}

\author{
Adelheid Weidinger 1,2 ${ }^{\mathbb{D}}$, Linda Birgisdóttir ${ }^{1,2,3} \mathbb{D}$, Julia Schäffer ${ }^{4}$, Andras T. Meszaros ${ }^{5} \mathbb{D}$, \\ Sergejs Zavadskis ${ }^{1,2}{ }^{\circ}$, Andrea Müllebner ${ }^{6}$, Matthias Hecker ${ }^{4}$, Johanna Catharina Duvigneau ${ }^{6}(\mathbb{D}$, \\ Natascha Sommer ${ }^{4}$ and Andrey V. Kozlov $1,2,7, * \mathbb{D}$
}

check for updates

Citation: Weidinger, A.; Birgisdóttir, L.; Schäffer, J.; Meszaros, A.T.; Zavadskis, S.; Müllebner, A.; Hecker, M.; Duvigneau, J.C.; Sommer, N.; Kozlov, A.V. Systemic Effects of mitoTEMPO upon

Lipopolysaccharide Challenge Are Due to Its Antioxidant Part, While Local Effects in the Lung Are Due to Triphenylphosphonium. Antioxidants 2022, 11, 323. https://doi.org/ $10.3390 /$ antiox 11020323

Academic Editor: Antonella Casola

Received: 30 December 2021

Accepted: 3 February 2022

Published: 6 February 2022

Publisher's Note: MDPI stays neutral with regard to jurisdictional claims in published maps and institutional affiliations.

Copyright: (C) 2022 by the authors. Licensee MDPI, Basel, Switzerland. This article is an open access article distributed under the terms and conditions of the Creative Commons Attribution (CC BY) license (https:// creativecommons.org/licenses/by/ $4.0 /)$.
1 Ludwig Boltzmann Institute for Traumatology, The Research Center in Cooperation with AUVA, 1200 Vienna, Austria; adelheid.weidinger@trauma.lbg.ac.at (A.W.); linda.birgisdottir@oulu.fi (L.B.); sergejs.zavadskis@trauma.lbg.ac.at (S.Z.)

2 Austrian Cluster for Tissue Regeneration, 1200 Vienna, Austria

3 Faculty of Biochemistry and Molecular Medicine, University of Oulu, 90570 Oulu, Finland

4 Department of Internal Medicine, Universities of Giessen and Marburg Lung Center (UGMLC), Member of the German Center for Lung Research (DZL), Excellence Cluster Cardio-Pulmonary Institute (CPI), Justus-Liebig University, 35392 Giessen, Germany; Julia.Schaeffer@innere.med.uni-giessen.de (J.S.); Matthias.hecker@innere.med.uni-giessen.de (M.H.); Natascha.Sommer@innere.med.uni-giessen.de (N.S.)

5 Department of Visceral, Transplant and Thoracic Surgery, Medical University of Innsbruck, 6020 Innsbruck, Austria; andras.meszaros@i-med.ac.at

6 Department for Biomedical Sciences, Institute for Medical Biochemistry, University of Veterinary Medicine, 1210 Vienna, Austria; Andrea.Muellebner@vetmeduni.ac.at (A.M.);

Catharina.Duvigneau@vetmeduni.ac.at (J.C.D.)

7 Laboratory of Navigational Redox Lipidomics, Department of Human Pathology, IM Seche-Nov Moscow State Medical University, 119146 Moscow, Russia

* Correspondence: andrey.kozlov@trauma.lbg.ac.at; Tel.: +43-(0)-5-93-93-41980

\begin{abstract}
Mitochondria-targeted antioxidants (mtAOX) are a promising treatment strategy against reactive oxygen species-induced damage. Reports about harmful effects of mtAOX lead to the question of whether these could be caused by the carrier molecule triphenylphosphonium (TPP). The aim of this study was to investigate the biological effects of the mtAOX mitoTEMPO, and TPP in a rat model of systemic inflammatory response. The inflammatory response was induced by lipopolysaccharide (LPS) injection. We show that mitoTEMPO reduced expression of inducible nitric oxide synthase in the liver, lowered blood levels of tissue damage markers such as liver damage markers (aspartate aminotransferase and alanine aminotransferase), kidney damage markers (urea and creatinine), and the general organ damage marker, lactate dehydrogenase. In contrast, TPP slightly, but not significantly, increased the LPS-induced effects. Surprisingly, both mitoTEMPO and TPP reduced the wet/dry ratio in the lung after $24 \mathrm{~h}$. In the isolated lung, both substances enhanced the increase in pulmonary arterial pressure induced by LPS observed within $3 \mathrm{~h}$ after LPS treatments but did not affect edema formation at this time. Our data suggest that beneficial effects of mitoTEMPO in organs are due to its antioxidant moiety (TEMPO), except for the lung where its effects are mediated by TPP.
\end{abstract}

Keywords: mitochondria-targeted antioxidants; mitoTEMPO; TPP; lipopolysaccharide; inflammatory response; lung; liver; pulmonary artery pressure; edema

\section{Introduction}

Understanding mechanisms that control intracellular signaling is an important but difficult task because signaling molecules, such as reactive oxygen species (ROS) are very short lived. Giant steps forward in understanding the role of mitochondrial ROS 
(mtROS) in physiological and pathological processes were made with the help of so-called mitochondria-targeted antioxidants (mtAOX). This class of molecules has two components. In the majority of cases, triphenylphosphonium (TPP) acts as the mitochondria-targeting moiety. TPP is a hydrophobic, positively charged molecule that selectively accumulates in mitochondria due to their unusually high membrane potential [1]. The second part of the molecule, the functional moiety comprising different types of antioxidants, is delivered to mitochondria and serves as a trap for ROS.

The effects of mtAOX, as described in the literature, are predominantly beneficial, mitigating pathological changes in the body [2-6]. However, there are also reports showing that the effect of mtAOX can be harmful [7]. The reason for this discrepancy is not clear, although recent data suggest that targeted antioxidants can act in different manners. Particularly, it has been shown that the carrier part of the molecule, in most cases TPP, can also manifest biological effects. For example, Powell and coauthors reported that intravenous injection of TPP in rats resulted in an increase in antioxidant activity in the liver, kidney, and spleen, but not in the lung [8]. In contrast, the antioxidant activity was decreased in the lung [8]. Interestingly, antioxidant activity did not correlate with levels of lipid peroxidation, which was increased in all organs [8]. Furthermore, it has been shown that different derivatives of TPP, which do not exert antioxidant properties, can substantially drop the rate of mitochondrial respiration linked to the major mitochondrial function of ATP synthesis [9]. It has also been shown that TPP molecules induce a significant increase in proton leak with a decrease in the mitochondrial membrane potential and an inhibition of respiratory chain complexes [10].

Indeed, it is known that a drop in mitochondrial membrane potential can be an additional mechanism that decreases the generation of mitochondrial ROS. For example, up-regulation of mitochondrial uncoupling proteins or treatment with ionophors such as carbonylcyanide-p-tri-fluoromethoxyphenylhydrazon can decrease the generation of mitochondrial ROS [11] and reduce ROS-mediated oxidative damage as well as ROS-mediated intracellular signaling [12]. Thus, theoretically, both an mtAOX such as mitoTEMPO, as well as its carrier molecule TPP alone, could reduce ROS production, either indirectly by decreasing mitochondrial membrane potential or directly by scavenging ROS.

There is a body of literature providing evidence for the involvement of ROS in the pathogenesis of pulmonary inflammation [13-15]. The most common way to induce an inflammatory response is the treatment with lipopolysaccharide (LPS). It has been shown that LPS stimulates ROS production in lung macrophages and endothelial cells [16] and that ROS increase endothelial permeability in the lung [16-18]. It has also been shown that pre-treatment with mitoTEMPO, a scavenger of mtROS, attenuated LPS-induced ROS generation and decreased lung endothelial permeability in an in vitro model [18].

The aim of this study was to compare the biological effects of TPP and mitoTEMPO in different organs in a rat model of the systemic inflammatory response syndrome (SIRS) induced by endotoxin in order to clarify which part of mitoTEMPO, TPP, or TEMPO is responsible for its effects.

\section{Materials and Methods}

\subsection{Chemicals}

All reagents were obtained from Merck/Sigma-Aldrich (Vienna, Austria) unless otherwise noted.

\subsection{Animals}

The experiments were performed on male Sprague-Dawley rats (weighing 350-400 g; $n$ is indicated in each figure legend; Charles River, Germany; Janvier Labs, France) which were kept under controlled standard animal housing conditions. The animals had free access to standard laboratory rodent food and water. They were kept for 7 days prior to usage in experiments for accommodation. 


\subsection{Lipopolysaccharide Treatment}

In the experiments described here, we used two lots of LPS from Escherichia coli serotype 026:B6 (activity $\geq 500000 \mathrm{EU} / \mathrm{mg}$ ); each was tested for its effect on the survival rate. The dose corresponding to a survival rate slightly above 50\% was selected (data not shown), which was $2.5 \mathrm{mg}$ and $1.75 \mathrm{mg}$ per $\mathrm{kg}$ body weight, respectively. LPS was dissolved in saline (Fresenius Kabi, Austria), and the LPS solution was vortexed for $1 \mathrm{~min}$ and sonicated for $30 \mathrm{~s}$ before application. Subsequently, the suspension was injected into the penis vein under isoflurane anesthesia in a volume ranging from 0.5 to $0.75 \mathrm{~mL}$. Normal saline was used as a control. For analgesia, Buprenorphine (Richter Pharma AG, Wels, Austria, $0.05 \mathrm{mg} / \mathrm{kg}$ body weight) was injected subcutaneously at the time of the LPS treatment and 8-10 h thereafter. For in vivo treatment, methyltriphenyl-phosphonium chloride (TPP, $50 \mathrm{nmol} / \mathrm{kg}$ ) or mitoTEMPO (50 nmol/ kg) was injected intraperitoneally one hour before the LPS/saline injection.

\subsection{Blood and Organ Sampling for Nitrite, Nitrogen Oxides, Organ Damage Marker and Gene Expression Measurements}

The experimental endpoint was $16 \mathrm{~h}$ after LPS/saline injection. Blood was collected in a lithium heparin tube from the tail vein before treatment and from the heart at $16 \mathrm{~h}$. Heparin tubes were centrifuged at $400 \times g$ for 10-15 min and plasma was collected. The rats were euthanized by injection of $2 \mathrm{~mL}$ thiopental-natrium and sodium carbonate (Thiopental, Rotexmedica $\mathrm{GmbH}$, Germany) under anesthesia, and organs were collected immediately into ice-cold Ringer solution (Fresenius Kabi, Germany), cut into small pieces, transferred to liquid nitrogen, and stored at $-80^{\circ} \mathrm{C}$ until further measurement.

\subsection{Animal Experiments, Sampling of Blood, Peritoneal and Bronchial Lavage for $\mathrm{H}_{2} \mathrm{O}_{2}$ Measurement}

The experimental model used for the measurement of $\mathrm{H}_{2} \mathrm{O}_{2}$ in different body compartments is illustrated in Figure 1a. At the end of the LPS treatment, rats were anesthetized by inhalation of a mixture of $3 \%$ isoflurane and oxygen. After a small skin cut, the left femoral artery was dissected and catheterized using a 24-gauge i.v. cannula (BD Neoflon, Becton Dickinson Infusion Therapy AB, Helsingborg, Sweden). Ten milliliters of whole blood was collected in a $50 \mathrm{~mL}$ Falcon tube prefilled with $200 \mu \mathrm{L}$ of sodium heparin (1000 IU/mL, Gilvasan Pharma GmbH, Vienna, Austria) for subsequent processing and for the in vitro part of the study. Following the blood sampling, the animals were euthanized by decapitation.

For bronchoalveolar lavage, we used a protocol adapted from [19]. Briefly, a 19-gauge needle hub was inserted into the trachea and $3 \mathrm{~mL}$ of ice-cold phosphate-buffered saline (PBS) and 10\% fetal bovine serum (FBS) were administered and aspirated slowly through the needle hub. The fluid was then transferred into a $15 \mathrm{~mL}$ tube (Greiner Bio-One, Austria) and kept on ice. The procedure was repeated four times.

To collect peritoneal cells, the peritoneum was exposed, and $5 \mathrm{~mL}$ of ice-cold PBS with 3\% FBS was injected into the peritoneal cavity through a needle, as described elsewhere [20]. A gentle massage to the abdomen was applied to dislodge any attached cells into the PBS solution. Then, the suspension was collected through a 22-gauge needle into a syringe and transferred into a $15 \mathrm{~mL}$ tube and kept on ice. The above-described procedure was repeated five times. 


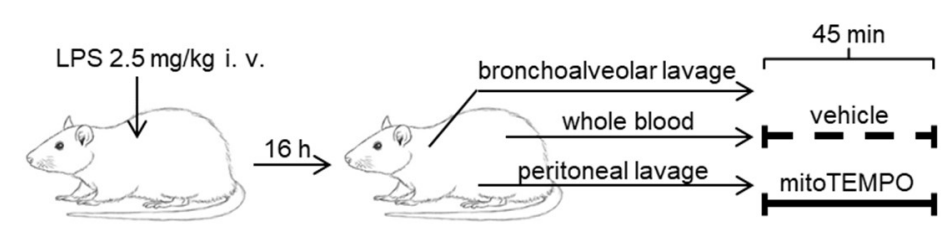

(a)

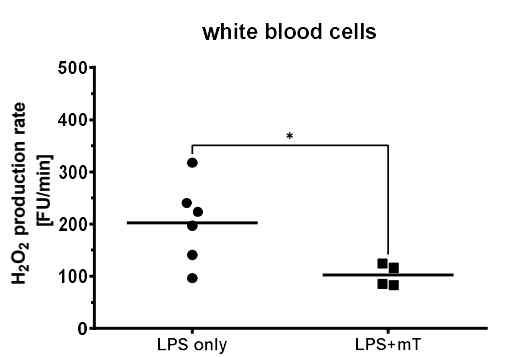

(c)

(d)

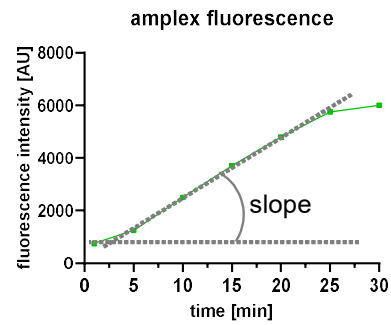

(b)

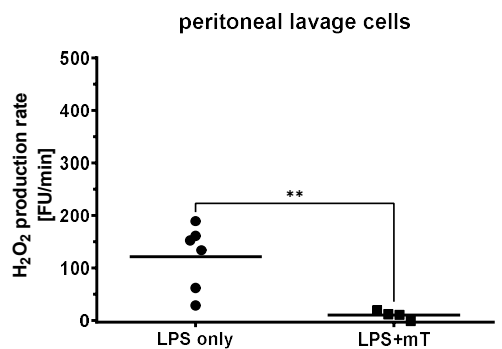

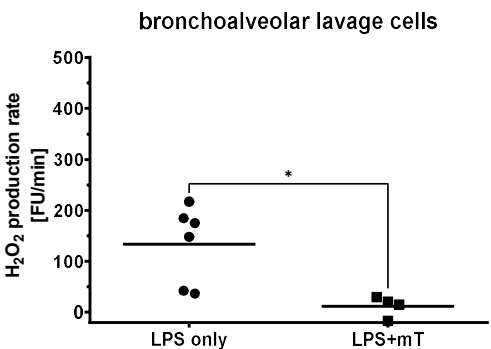

(e)

Figure 1. Effect of mitoTEMPO (mT) on generation of ROS in different body compartments of rats. (a) Rats were challenged with $2.5 \mathrm{mg} / \mathrm{kg}$ body weight lipopolysaccharide (LPS) and biological fluids were withdrawn after $16 \mathrm{~h}$. (b) Scheme of $\mathrm{H}_{2} \mathrm{O}_{2}$ production rate assessed by Amplex Red by calculating the slope. (c-e) Effect of mitoTEMPO on $\mathrm{H}_{2} \mathrm{O}_{2}$ production rate of white blood cells (c), peritoneal lavage (d), and bronchoalveolar lavage (e). Unpaired $t$-test, ${ }^{*} p<0.05,{ }^{* *} p<0.01$.

\subsubsection{Purification of Cells and Incubation with mitoTEMPO}

The suspension of bronchoalveolar and peritoneal cells was centrifuged at $400 \times g$ for $10 \mathrm{~min}$ at $4{ }^{\circ} \mathrm{C}$, the supernatant was discarded, and the cell number was counted with a Cell-Dyn 3700 Hematology Analyzer (Abbott Laboratories, Lake Bluff, IL, USA). After a complete blood count, blood samples were centrifuged at $300 \times g$ for $10 \mathrm{~min}$ at $4{ }^{\circ} \mathrm{C}$, then the plasma and buffy coat were removed and the remaining blood pellet was incubated with a lysis buffer containing $168 \mathrm{mM} \mathrm{NH}_{4} \mathrm{Cl}, 10 \mathrm{mM} \mathrm{KHCO}_{3}$, and $973 \mu \mathrm{M}$ EDTA for 10 min at $4{ }^{\circ} \mathrm{C}$. Cells were then washed twice with PBS at $10{ }^{\circ} \mathrm{C}$ followed by centrifugation at $400 \times g$ for $8 \mathrm{~min}$ and finally, counts were performed. For the in vitro mtAOX treatment, cell suspensions were incubated at $37^{\circ} \mathrm{C}$ for $45 \mathrm{~min}$ with mitoTEMPO (500 nM) or with vehicle $(\mathrm{NaCl})$.

\subsubsection{Extracellular $\mathrm{H}_{2} \mathrm{O}_{2}$ Measurement}

The $\mathrm{H}_{2} \mathrm{O}_{2}$ production in the cell suspension was assessed by $\mathrm{N}$-acetyl-3,7-dihydroxy phenoxazine (Amplex Red, Life Technologies, Eugene, OR, USA). Amplex Red is a sensitive and chemically stable fluorogenic probe and produces the fluorescent resolufin with $\mathrm{H}_{2} \mathrm{O}_{2}$ in a horseradish peroxidase (HRP)-catalyzed oxidation, with excitation/emission maxima at 563/587. Reaction stoichiometry of Amplex Red and $\mathrm{H}_{2} \mathrm{O}_{2}$ is 1:1 [21]. Using a fluorescence plate reader (POLARstar Omega 3MG, Labtech, Germany), white blood cells, bronchoalveolar lavage, and peritoneal lavage cells $\left(15 \times 10^{3}\right.$ cells per well in Krebs buffer $)$ were incubated in black 96-well microplates (Greiner Cellstar) at $37^{\circ} \mathrm{C}$ with Amplex Red $(10 \mu \mathrm{M})$ and $\mathrm{HRP}\left(0.2 \mathrm{U} \mathrm{mL}^{-1}\right)$. The fluorescence intensity was recorded for $30 \mathrm{~min}$ with an excitation of $544 \mathrm{~nm} /$ emission of $590 \mathrm{~nm}$ and gain at 1200. The slope was calculated from a 10 min interval between the 5 th and 15th min of the measurement nm (Figure $1 \mathrm{~b}$ ).

\subsection{Nitrite and Nitrogen Oxides Measurement}

Nitric oxide (NO) was measured in duplicates from plasma samples with the Sievers 280i-NO Analyzer (General Electric's Analytical Instruments, Boulder, USA). Prior to measuring, a calibration curve was recorded using a standard stock solution $(0.069 \mathrm{~g}$ 


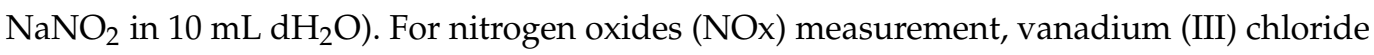
was applied $\left(0.2 \mathrm{~g} \mathrm{~V}(\mathrm{III}) \mathrm{Cl}_{3}\right.$ in $25 \mathrm{~mL}$ of $1 \mathrm{M}$ hydrochloride acid $\left.(\mathrm{HCl})\right)$ as a reactive agent in a $95{ }^{\circ} \mathrm{C}$ water bath (Grant Instruments, Beaver Falls, USA). For nitrite $\left(\mathrm{NO}_{2}{ }^{-}\right)$measurement, $50-100 \mu \mathrm{L}$ sodium iodide $\left(0.05 \mathrm{~g} \mathrm{NaI}\right.$ in $0.5 \mathrm{~mL} \mathrm{dH}_{2} \mathrm{O}$ supplemented with $5 \mathrm{~mL}$ acetic acid) was applied as a reactive agent.

\subsection{Determination of Heme Oxygenase Activity}

Liver tissue was homogenized using a Potter-Elvehjem with PTFE pestle on ice in 1:10 weight to volume ratio in a Tris buffer $(20 \mathrm{mM})$ containing $300 \mathrm{mM}$ sucrose and $8 \mu \mathrm{M}$ EDTA (pH 7.4). Samples were distributed in aliquots of $300 \mu \mathrm{L}$ and snap frozen in liquid nitrogen until activity measurement. The residual homogenate was used to constitute a pool, which was used as an internal standard (IS). For the determination of HO activity, a coupled enzyme assay was used, which determines the end-product bilirubin (BR). For each sample, which was analyzed in duplicates, $25 \mu \mathrm{L}$ of homogenate was added to ice-cold $92 \mu \mathrm{L} \mathrm{HO}-$ assay buffer $\left(\mathrm{KH}_{2} \mathrm{PO}_{4}(25 \mathrm{mM})\right.$ and $\mathrm{K}_{2} \mathrm{HPO}_{4}(75 \mathrm{mM})(\mathrm{pH} 7.4), 8 \mu \mathrm{L}$ deferoxamine (DFO, $100 \mathrm{mM}$, Desferal ${ }^{\circledR}$, Novartis, Switzerland), $4 \mu \mathrm{L}$ hemin $(5 \mathrm{mM}$, Fluka, Buchs, Switzerland), supplemented with $25 \mu \mathrm{L}$ NADPH (10 mM, Sigma). For each sample, one control was included containing $4 \mu \mathrm{L}$ DMSO instead of hemin and $25 \mu \mathrm{L}$ HO-assay buffer instead of NADPH. Samples were vortexed and incubated for $30 \mathrm{~min}$ at $37^{\circ} \mathrm{C}$ under constant agitation. The reaction was stopped by transferring the samples on ice. BR was extracted into benzene as described previously [22]. BR concentration in extracts was determined using a double beam spectrophotometer (U-3900, Hitachi, Tokyo, Japan) and a BR standard calibration curve. The detection limit of BR using this method was determined as 5 pmol BR. We found that the biliverdin reductase activity (the capacity to convert biliverdin into $\mathrm{BR}$ ) in liver tissue was much higher ( $<10$ times, data not shown) than that of $\mathrm{HO}$, indicating that BVR activity is not limiting and BV formed by the $\mathrm{HO}$ enzyme is completely reduced to BR by the underlying BVR. The amount of BR determined in samples was corrected for the BR amount determined in the negative controls and corrected for the underlying protein content. Protein determination was performed using the Bradford method and a BSA calibration curve, as described earlier [22]. Enzyme activities were expressed as nmol $\mathrm{BR}$ formed per $\mathrm{mg}$ protein in $30 \mathrm{~min}$.

\subsection{Gene Expression of Inflammatory and Cell Stress Markers, iNOS, HO-1, IL6, TNFR1, NFkBia and $\mathrm{CHOP}$}

Gene expression analysis was performed using qPCR as described elsewhere [23]. RNA was isolated from $100 \mu \mathrm{L}$ liver homogenate prepared as described above using 1 $\mathrm{mL}$ of TriReagent ${ }^{\mathrm{TM}}$ (MRC, Cincinnati, OH, USA). Extraction of RNA was performed according to the manufacturer's protocol. The amount of extracted RNA was determined spectrophotometrically at $260 \mathrm{~nm}$ and purity was assessed by the 260/280 $\mathrm{nm}$ ratio on an Eppendorf BioPhotometer plusUV/VIS (Eppendorf, Hamburg, Germany). Copy DNA was prepared as previously described [23]. Equal aliquots from each cDNA were pooled to generate an internal standard (IS) which was used as a reference for the quantification. Primer pairs used for the expression analysis of targets and the internal reference genes, hypoxanthine ribosyltransferase (HPRT), and cyclophilin A (Cyc) are described in detail in Table A1. PCR reactions contained $\mathrm{iTaq}^{\mathrm{TM}} \mathrm{DNA}$ polymerase ${ }^{\mathrm{TM}}(0.625 \mathrm{U} /$ reaction; BioRad $)$, primers (250 nmol/L each, Invitrogen), dNTP at a final concentration of $200 \mu \mathrm{mol} / \mathrm{L}$ (each), $3 \mathrm{mmol} / \mathrm{L} \mathrm{MgCl}_{2}$ (except for IL-6, $2 \mathrm{mmol} / \mathrm{L}$; iNOS, $1.5 \mathrm{mmol} / \mathrm{L}$ ), and SYBR ${ }^{\circledR}$ green I as reporter dye $(0.5 \times)$ in the provided reaction buffer at a final volume of $12 \mu \mathrm{L}$. The qPCR was carried out on a CFX96 ${ }^{\mathrm{TM}}$ (Bio-Rad, Hercules, CA, USA) real-time PCR detection system. Data were analyzed using the inbuilt software CFX manager (Version 2.0, Bio-Rad) in the linear regression mode. A modified comparative $\Delta \Delta \mathrm{Cq}$ method [24] was applied for data analyses. All data were normalized for the reference genes Cyclophilin A and hypoxanthinribosyl transferase (HPRT) and calculated as fold change relative to the IS. 


\subsection{Organ Damage Markers}

Plasma levels of aspartate aminotransferase (AST), alanine aminotransferase (ALT), lactate dehydrogenase (LDH), urea, creatinine (crea), and creatinine kinase (CK) were analyzed with the Roche Cobas cIII analyzer (Roche, Basel, Switzerland) according to the manufacturer's instruction.

\subsection{Wet/Dry Ratio}

Approximately $0.2-0.3 \mathrm{~g}$ of lung tissue was used to determine the wet/dry ratio. The tissue was weighted before and after freeze drying (Christ Alpha 1-2 LD plus, Osterode am Harz, Germany). A program of $-49.6{ }^{\circ} \mathrm{C}$ and vacuum of $0.63 \mathrm{mbar}$ was operated for approximately $18 \mathrm{~h}$. The ratio between wet and dry weight of the lung tissue was calculated.

\subsection{Isolated Perfused and Ventilated Lungs}

Isolated perfused and ventilated lungs were prepared as previously described [25]. Briefly, male C57BL/6J mice were deeply anesthetized by an intraperitoneal injection of ketamine $(100 \mathrm{mg} / \mathrm{kg}$ bodyweight $(\mathrm{BW}))$ and xylazine $(20 \mathrm{mg} / \mathrm{kg}$ BW) with heparin (50,000 I.U. $/ \mathrm{kg} \mathrm{BW)} \mathrm{as} \mathrm{an} \mathrm{anticoagulant.} \mathrm{Mice} \mathrm{were} \mathrm{intubated} \mathrm{via} \mathrm{a} \mathrm{tracheostoma} \mathrm{and}$ ventilated with normoxic gas $\left(21 \% \mathrm{O}_{2}\right.$, positive pressure ventilation, $10 \mu \mathrm{L} / \mathrm{g} \mathrm{BW}$ tidal volume, 90 breath/min, and $3 \mathrm{~cm} \mathrm{H}_{2} \mathrm{O}$ positive end-expiratory pressure). After opening the chest, catheters were inserted in the pulmonary artery and the left atrium and perfused with Krebs-Henseleit buffer (Serag Wiessner, Naila, Germany) using a peristaltic pump (ISM834A V2.10, Ismatec) to reach a final flow of $2 \mathrm{~mL} / \mathrm{min}$. In parallel with the onset of artificial perfusion, ventilation was changed from ambient air to a pre-mixed gas $\left(21 \% \mathrm{O}_{2}, 5.3 \% \mathrm{CO}_{2}\right.$, balanced with $\left.\mathrm{N}_{2}\right)$. The lungs were removed from the thorax and freely suspended on a force transducer to monitor organ weight. After reaching a stable state, $6 \mu \mathrm{g} / \mathrm{mL}$ (final concentration) of lipopolysaccharide (LPS, Samonella enterica serotype abortus equi) was added to the perfusate and recirculated for $2 \mathrm{~h}$, followed by application of mitoTEMPO/TPP or the solvent as control each $10 \mathrm{~min}$ (minute: 145, 155, 165, 175) in increasing concentrations (final concentrations: $0.1,0.5,1.0,10 \mu \mathrm{M}$ ). The pulmonary arterial pressure (PAP) and lung weight (LW) were monitored continuously during the experiment. Changes in PAP $(\triangle \mathrm{PAP})$ and lung weight $(\triangle \mathrm{LW})$ were determined by calculating the difference in the respective value averaged from minute 9 to 10 after mitoTEMPO, TPP, or solvent application and the value averaged over one minute before mitoTEMPO, TPP, or solvent application.

\subsection{Statistical Analysis}

Data were analyzed using GraphPad-Prism software (8.0.1.244, USA) by unpaired t-test, one-way analysis of variance (ANOVA) followed by Fisher's least significant difference test, or by two-way ANOVA with Tukey's post hoc test. In all tests, $n$ (sample size) represents biological replicates (donors). The statistical tests and the $n$ number are indicated in the figure legends. Results are presented as the mean \pm SEM. Level of significance was set at 0.05 and is indicated as ${ }^{*} p<0.05,{ }^{* *} p<0.01,{ }^{* * *} p<0.001,{ }^{* * * *} p<0.0001$.

\section{Results}

Immune cells, particularly macrophages and monocytes, are the first components of the innate immune system responding to bacterial toxins by the elevated generation of ROS. Therefore, we first tested whether mitoTEMPO is able to inhibit ROS generation in different body compartments in our model. We analyzed the ROS $\left(\mathrm{H}_{2} \mathrm{O}_{2}\right)$ production capacity of immune cells derived from three compartments, namely blood (white blood cells (WBC) isolated from the blood), peritoneal lavage, and bronchoalveolar lavage from animals treated with LPS in vivo (Figure 1a). To avoid a complex procedure of normalization, we tested the response of the cells within the biological compartments to mitoTEMPO ex vivo (Figure 1a). The corresponding cell preparations were divided into two aliquots; one was incubated with saline, the other one with mitoTEMPO. The results show that mitoTEMPO 
efficiently decreases ROS generation in cells from all three compartments (Figure 1c-e), indicating that mitochondrial ROS-mediated pathways are operating in our model. Since not only macrophages but also other lung cells may generate ROS, we also confirmed these findings in precision-cut lung slices (PCLS, Supplementary Materials S1). PCLS contain all lung cell types and maintain tissue structure but are not influenced by systemic factors and are therefore suitable to investigate local effects in the tissue. We showed that incubation with LPS increases cytoplasmic ROS and both TPP and mitoTEMPO attenuate ROS levels (Supplementary Figure S1), suggesting that a part of cytoplasmic ROS is released from mitochondria. In addition, ROS from non-mitochondrial sources could also play a role, which could be attenuated by non-targeted antioxidant TEMPO [26]. In our study, we did not consider TEMPO as an appropriate control, because, as mentioned, it accumulates in all cell compartments and can exert non-mitochondrial effects. Since both TPP and mitoTEMPO accumulate and act in mitochondria, we studied separately effects of both these molecules in the following experiments.

One of the characteristic features of the inflammatory response in the endotoxic shock model is the upregulation of inducible nitric oxide synthase (iNOS) and excessive formation of nitric oxide (NO). Therefore, we investigated the effects of TPP and mitoTEMPO on the levels of LPS-induced NO metabolites in blood plasma. As carbon monoxide generated by heme oxygenase $(\mathrm{HO})$ could, similarly to $\mathrm{NO}$, exert biological activities on mitochondria, we also determined $\mathrm{HO}$ activity in liver tissue. As expected, $\mathrm{HO}$ activity showed similar kinetics to all NO derivatives (Figure 2). Both nitrite and NOx as well as HO activity were elevated upon LPS treatment, and significantly attenuated by mitoTEMPO compared to the LPS + TPP group (Figure 2a-c). Interestingly, TPP slightly increased the mean values of all these parameters, but this increase was significant only for the plasma levels of nitrite. These data indicate that the beneficial effects of mitoTEMPO can only be achieved by its antioxidant moiety.

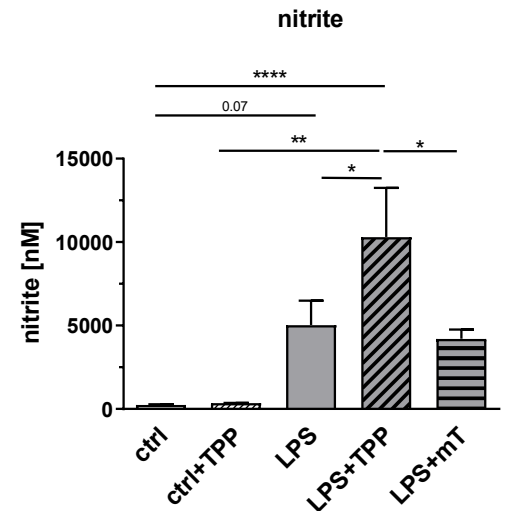

(a)

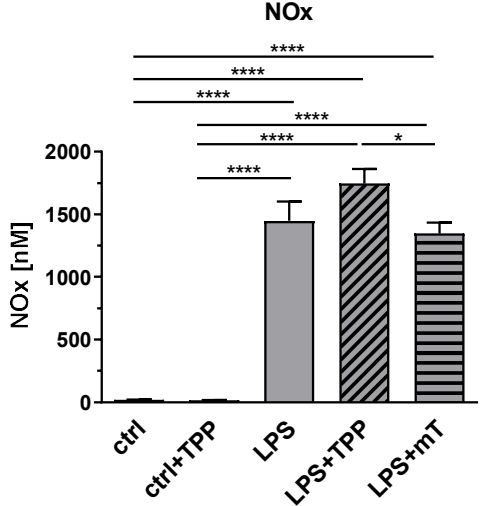

(b)

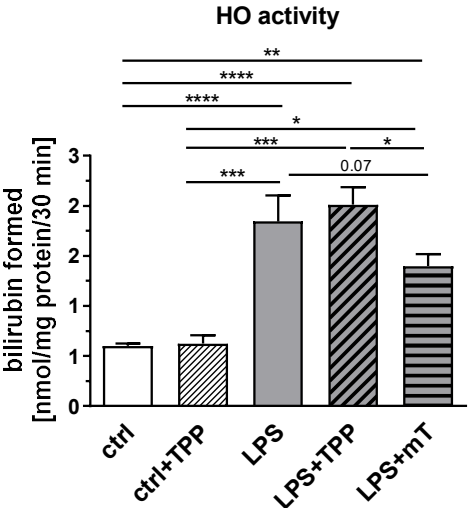

(c)

Figure 2. Effect of lipopolysaccharide (LPS, $1.75 \mathrm{mg} / \mathrm{kg}$ ), methyltriphenylphosphonium chloride (TPP, $50 \mathrm{nmol} / \mathrm{kg}$ ) and mitoTEMPO (mT, $50 \mathrm{nmol} / \mathrm{kg}$ ) on the levels of nitric oxide (NO) derivatives (a), and nitrite and nitrogen oxide (NOx) in rat plasma (b), as well as heme oxygenase (HO) activity in liver tissue (c). MitoTEMPO decreases the activity of the tested metabolites $(\mathbf{a}-\mathbf{c})$ while TPP increases the mean values of $\mathrm{NO}$ derivatives (a) and NOx (b) in the blood. One-way analysis of variance (ANOVA), Fisher's least significant difference (LSD), $n=3-8$, mean \pm SEM, ${ }^{*} p<0.05,{ }^{* *} p<0.01$, *** $p<0.001, * * * * * 0.0001$.

The liver plays an important physiological role in LPS detoxification and, in particular, hepatocytes are involved in the clearance of endotoxin of intestinal derivation [27]. Therefore, we measured the effect of mitoTEMPO on the inflammatory response in the liver. We determined the mRNA expression levels of iNOS and HO-1, as well as other inflammation-associated response genes, such as interleukin (IL)6, tumor necrosis factor 
receptor (TNFR)1, nuclear factor kappa B inhibitor alpha (NFkBia), and CCAAT/enhancerbinding protein homologous protein (CHOP) (Figure 3). We observed a clear response of all markers to the LPS challenge; their expression levels were increased (Figure 3). The treatment with mitoTEMPO decreased the mean values of iNOS and IL-6 (Figure 3a,c), whereas the treatment with TPP even further increased the gene expression levels, with that of IL-6 being significant. These data are in line with the previously reported data on beneficial effects of mtAOX such as mitoTEMPO [2-6]. Interestingly, we found that the LPS-mediated increase in the expression levels of TNFR1, NFkBialpha, and CHOP was not affected by mitoTEMPO, indicating that mainly the pathogen-associated molecules drive these targets. Except for CHOP, we found no down-modulating effect of TPP on the expression of target genes in the liver.

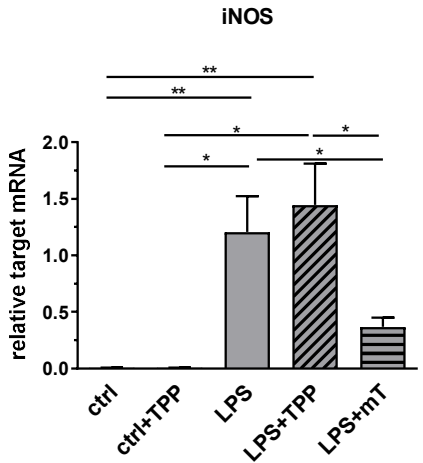

(a)

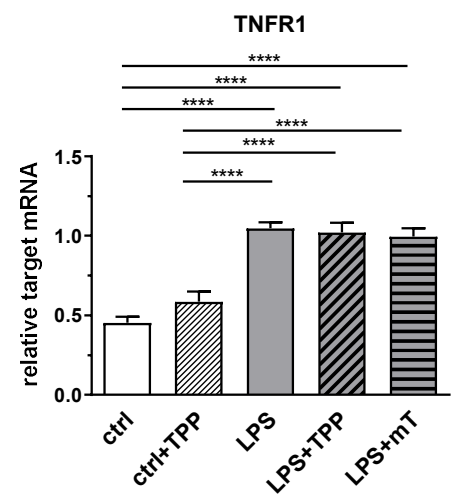

(d)

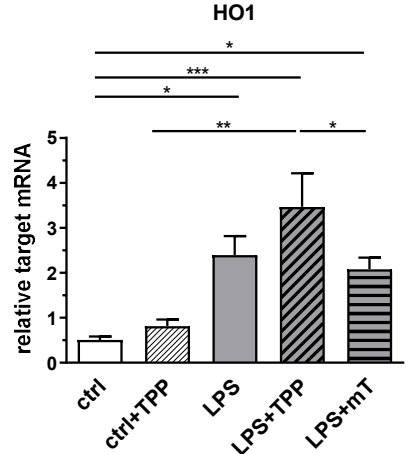

(b)

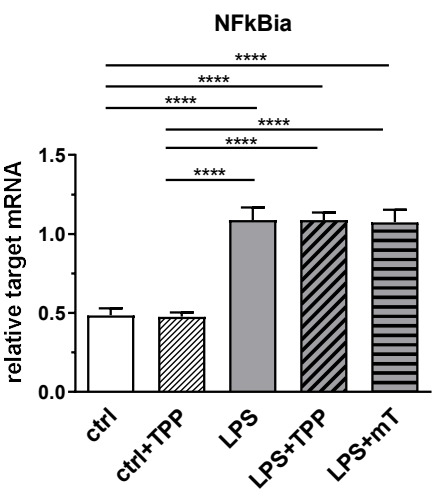

(e)

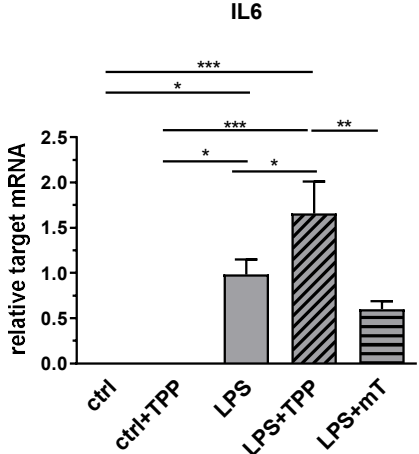

(c)

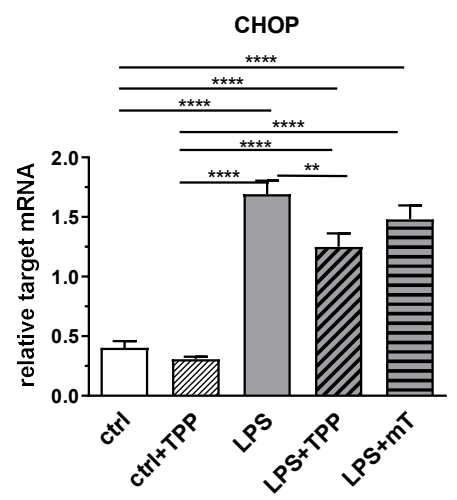

$(\mathbf{f})$

Figure 3. Effect of lipopolysaccharide (LPS, $1.75 \mathrm{mg} / \mathrm{kg}$ ), methyltriphenylphosphonium chloride (TPP, $50 \mathrm{nmol} / \mathrm{kg}$ ) and mitoTEMPO (mT, $50 \mathrm{nmol} / \mathrm{kg}$ ) on gene expression of iNOS (a), HO1 (b), IL-6 (c), TNFR1 (d), NFkBia (e), and CHOP (f) in rat liver. After LPS injection, gene expression levels of all mediators increased (a-f). MitoTEMPO treatment decreased the mean values of iNOS (a) and IL-6 (c). An increase is seen in IL-6 expression in the LPS+TPP group compared to the other LPS groups. All data are normalized for the expression levels of the reference genes (cyclophilin A and hypoxanthine-guanine phosphoribosyl transferase (HPRT)) and expressed as fold changes relative to the internal standard. ${ }^{*} p<0.05,{ }^{* *} p<0.01,{ }^{* * *} p<0.001$. One-way analysis of variance (ANOVA), Fisher's least significant difference (LSD), $n=3-8$, mean $\pm \mathrm{SEM}^{*} p<0.05,{ }^{* *} p<0.01,{ }^{* * *} p<0.001$, **** $p<0.0001$. Abbreviations: inducible nitric oxide synthase (iNOS), heme oxygenase 1 (HO- 1 ), interleukin 6 (IL-6), tumor necrosis factor receptor 1 (TNF-R1), nuclear factor kappa B inhibitor alpha (NFkBia), CCAAT/enhancer-binding protein homologous protein (CHOP).

Next, we investigated the effect of mitoTEMPO on tissue damage markers. MitoTEMPO significantly decreased the levels of the liver damage markers AST (Figure 4a) and ALT (Figure $4 \mathrm{~b}$ ), the organ damage marker LDH (Figure $4 \mathrm{c}$ ), and the markers for 
kidney dysfunction and damage, urea (Figure 4d) and creatinine (Figure 4e) in plasma. All these markers showed a similar response, as we found for the expression of iNOS, HO-1, and IL-6 in the liver. Accordingly, TPP presence also showed no beneficial effect on these markers (Figure $4 \mathrm{a}-\mathrm{f}$ ). Our data indicate that mitochondrial ROS contribute, to a significant extent, to the LPS-triggered increase in the levels of liver and kidney damage markers and the hepatic expression of iNOS, $\mathrm{HO}-1$, and IL-6, since the antioxidant moiety of mitoTEMPO was capable of counteracting their rise.

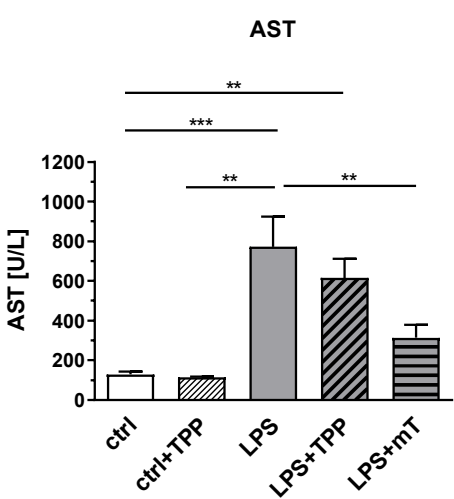

(a)

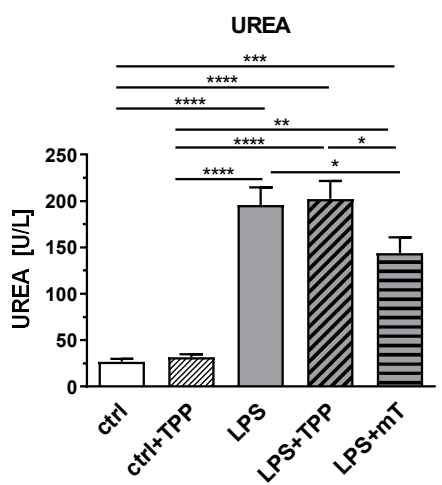

(d)

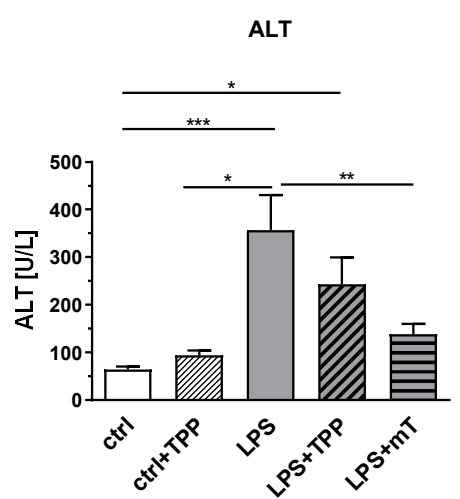

(b)

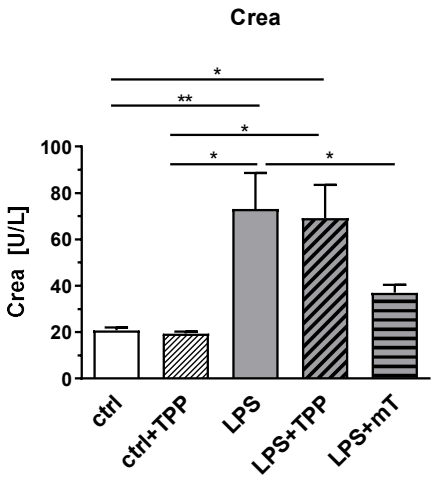

(e)

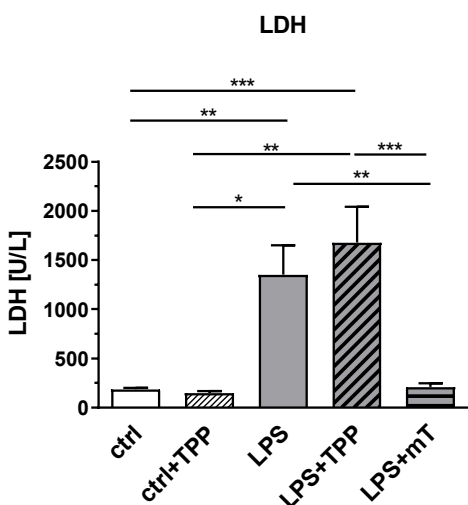

(c)

CK

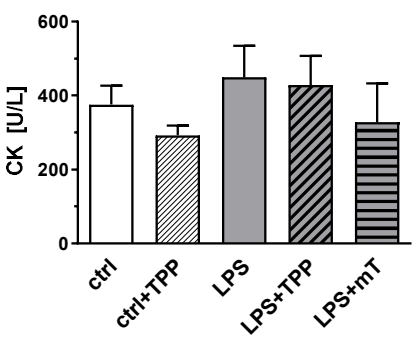

(f)

Figure 4. Effect of lipopolysaccharide (LPS, $1.75 \mathrm{mg} / \mathrm{kg}$ ), methyltriphenylphosphonium chloride $(\mathrm{TPP}, 50 \mathrm{nmol} / \mathrm{kg})$ and mitoTEMPO $(\mathrm{mT}, 50 \mathrm{nmol} / \mathrm{kg})$ on organ damage markers. An increase for few values is seen in the LPS + TPP group compared to the LPS group for all tissue damage markers. LDH showed significant difference between the LPS + TPP and all other groups. The LPS + mitoTEMPO seems to indicate beneficial effects of antioxidants (AOX) being lower than both the LPS and the LPS + TPP groups for all values measured; (a) aspartate aminotransferase (AST), (b) alanine aminotransferase (ALT), (c) lactate dehydrogenase (LDH), (d) urea, (e) creatinine (Crea) and (f) creatine kinase (CK). One-way analysis of variance (ANOVA), Fisher's least significant difference (LSD), $n=3-8$, mean $\pm \mathrm{SEM}^{*} p<0.05,{ }^{* *} p<0.01,{ }^{* * *} p<0.001,{ }^{* * * *} p<0.0001$.

Surprisingly, we observed a different picture in the lung. To estimate LPS-mediated damage, we determined the wet/dry ratio (W/D ratio) of lung tissue. LPS substantially elevated the W/D ratio, reflecting lung edema and serious lung damage (Figure 5). The application of mitoTEMPO and TPP showed that both compounds reduced the W/D ratio to a similar extent (Figure 5). This phenomenon led us to the assumption that it is the carrier molecule TPP that is responsible for the reduction of the W/D ratio in the lung and not the antioxidant part of mitoTEMPO.

This led us to a more profound investigation of mitoTEMPO/TPP action in the lung. To better dissect the effects of mitoTEMPO and TPP, we performed experiments in isolated lungs (ex vivo). Surprisingly, both mitoTEMPO and TPP strongly elevated PAP in LPS- 
treated isolated lungs, and no difference was observed between the effects of mitoTEMPO and TPP (Figure $6 a, b)$. However, edema formation was not affected by the application of mitoTEMPO or TPP (Figure $6 c, d$ ). These findings support the notion that mitoTEMPO and TPP similarly affect the lung and that the increase in PAP and the development of edema have different mitoTEMPO/TPP-mediated regulatory mechanisms.

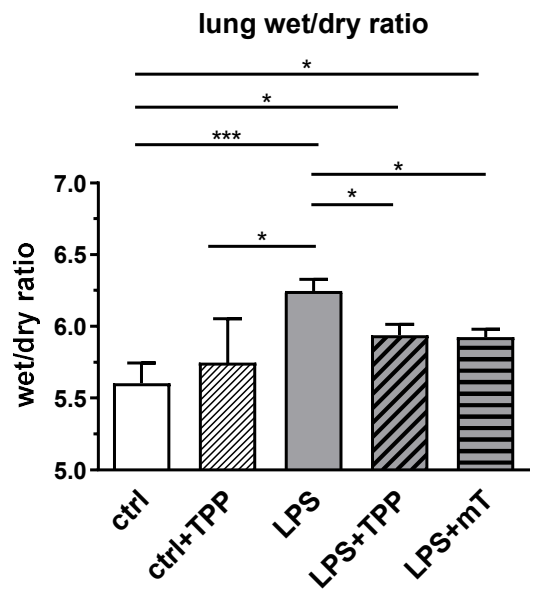

Figure 5. Effect of lipopolysaccharide (LPS, $1.75 \mathrm{mg} / \mathrm{kg}$ ), methyltriphenylphosphonium chloride $(\mathrm{TPP}, 50 \mathrm{nmol} / \mathrm{kg})$ and mitoTEMPO $(\mathrm{mT}, 50 \mathrm{nmol} / \mathrm{kg})$ on the wet/dry ratio in rat lung. Treatment with lipopolysaccharide (LPS) elevated the wet/dry ratio whereas both TPP and mitoTEMPO reduced the wet/dry ratio to a similar extent. One-way analysis of variance (ANOVA), Fisher's least significant difference (LSD), $n=3-8$, mean \pm SEM, ${ }^{*} p<0.05,{ }^{* * *} p<0.001$.

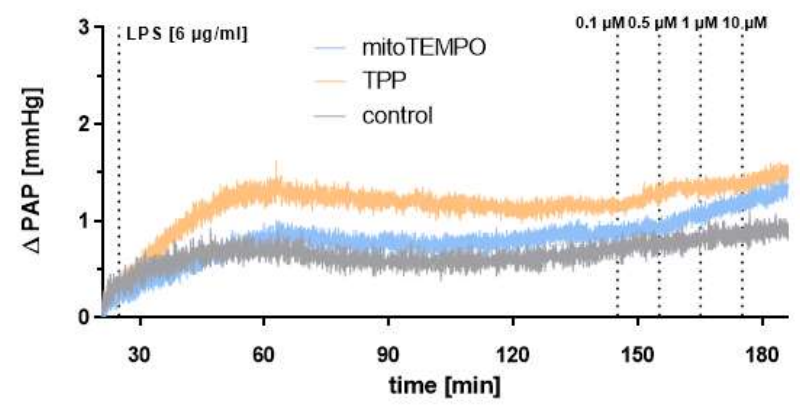

(a)

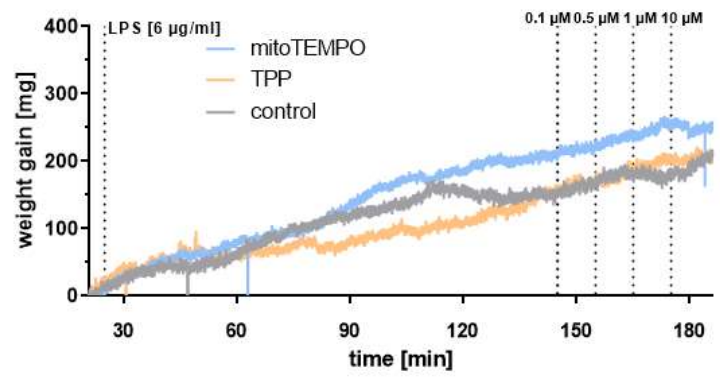

(c)

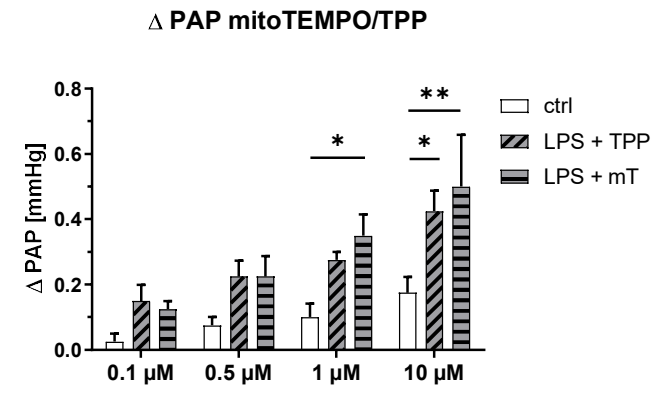

(b)

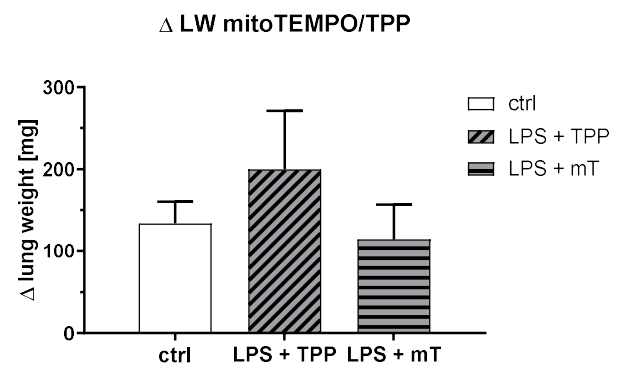

(d)

Figure 6. Isolated blood free perfused lung. Pulmonary arterial pressure (PAP) and lung weight (LW) in response to lipopolysaccharide (LPS, $6 \mu \mathrm{g} / \mathrm{mL}$ ), methyltriphenylphosphonium chloride (TPP), and mitoTEMPO (mT) treatment. Course of PAP increase (a) or weight gain (c) after the start of the experiment (mean values of $n=4$ isolated lungs each experiment). PAP increase (b) after repetitive application of mitoTEMPO/TPP and weight gain (d) after the last application of mitoTEMPO/TPP. Two-way analysis of variance (ANOVA) with Tukey's post hoc test, $n=4$ each group, mean $\pm \mathrm{SEM},{ }^{*} p<0.05$, ** $p<0.01$. 


\section{Discussion}

Here, we show that the effects of TPP and mitoTEMPO differ in the lung from those elicited in the other organs. We observed that markers of organ damage as well as markers of the inflammatory response, particularly those associated with $\mathrm{NO}$ metabolism that increase in circulating blood, can be significantly lowered by mitoTEMPO, but not by TPP, pointing towards the role of ROS in the upregulation of theses markers in response to LPS. The effect of mitoTEMPO is in line with previous reports published by us and others in similar models of acute inflammation [2-6]. In contrast, both TPP and mitoTEMPO reduced the $\mathrm{W} / \mathrm{D}$ ratio of the lung $24 \mathrm{~h}$ after LPS treatment to a similar extent. Moreover, both similarly elevated pulmonary arterial pressure (PAP) in isolated lung within $3 \mathrm{~h}$ after LPS treatment. The elevated PAP determined in isolated lung, however, was not accompanied by enhanced pulmonary edema formation. In general, this discrepancy between data obtained in the lung and other organs suggests that the TPP part is responsible for the effects in the lung and the TEMPO part for the effects in the rest of the body determined at the later time points. We do not know exactly how TPP acts in the lung, but there are several explanations based on the published data.

A single injection of LPS almost immediately elevates PAP, which drops after a couple of hours [28]. Lung edema in rats subjected to LPS starts at 6-8 h, reaching a maximum by $24 \mathrm{~h}$ [29]. These observations support the assumption that there are different mechanisms regulating PAP and edema in the endotoxic shock model.

The increase in PAP in response to LPS has been associated with the release of vasoconstrictors [28]. This is a result of the direct interaction of LPS with endothelial cells via toll-like receptor (TLR) 4 which activates the release of intracellular ROS [30]. A number of vasoactive substances are potentially capable of regulating PAP. One of the most prominent is endothelin 1 (ET-1), which is released by endothelial cells and regulated by ROS. ET-1 is activated by the endothelin-converting enzyme (ECE). This activation is regulated by ROS; superoxide radicals $\left(\mathrm{O}_{2}{ }^{\bullet-}\right)$ can inhibit endothelin-converting enzyme activity (ECE) and decrease ET-1 synthesis [31]. Thus, scavenging of $\mathrm{O}_{2}{ }^{\bullet-}$ can activate ECE and increase PAP. This is in line with the effect of mitoTEMPO/TPP on PAP, if we consider that the reduction in membrane potential has a stronger impact on lowering the ROS level than scavenging of ROS. This assumption is supported by our recent observation on inhibition of ROS formation by TPP in pulmonary smooth muscle cells [32]. The development of LPS-induced edema is very often associated with elevated PAP. In our experiments, both mitoTEMPO and TPP decreased the W/D ratio in vivo and did not affect weight gain in the isolated lung, but elevated PAP. We assume that the development of lung edema and the increase in PAP in the isolated lung are regulated by different mechanisms. First, they occur at different time points. In response to LPS, we observed a continuous increase in weight in the isolated lung, while the PAP increased strongly at the very beginning of LPS treatment. Second, mitoTEMPO/TPP exerted different effects on PAP and weight gain.

In addition, although the mechanisms increasing PAP and inducing edema are different, they have a common feature being activated by ROS. We assumed that similar effects of mitoTEMPO and TPP occurred in the lung, because both modulate ROS production in a similar manner. Since increased ROS levels in response to LPS can originate not only from macrophages but also from epithelial cells, we examined this assumption in PCLS by means of laser scanning microscopy. PCLS comprise all types of lung cells and therefore, we expected that we can test our assumption in this experimental model. Indeed, as expected, we observed a drastic increase in ROS levels in PCLS in response to LPS and both TPP and mitoTEMPO abolished this effect. This supports our assumption that ROS are involved in the regulation of PAP and edema at early and late phases of the inflammatory response, respectively.

Indeed, ROS affect alveolar amiloride-sensitive epithelial sodium channels (ENaCs), which play a crucial role in sodium transport and fluid reabsorption in the lung [33]. LPS, frequently employed to induce acute liver injury in experimental animal models, has been reported to regulate $\mathrm{ENaC}$ expression and alveolar fluid clearance. $\mathrm{ENaC}$ has 
a critical role in the clearance of edema fluid from the alveolar space. Several lines of evidence suggest that protein kinase $\mathrm{C}$ (PKC) is likely to play a permissive role in hydrogen peroxide $\left(\mathrm{H}_{2} \mathrm{O}_{2}\right)$-mediated endothelial permeability and lung edema [34,35]. It seems that ROS released by macrophages are involved in edema induction. Reduction of endotoxininduced macrophage activation results in the reduction of lung edema and mortality in vivo [36]. Furthermore, it has even been shown that $\mathrm{H}_{2} \mathrm{O}_{2}$ may enter the mesenteric lymph and travel to the lung, where it induces edema [37]. All these data suggest that the development of pulmonary edema in vivo is largely affected by systemic activation of the immune system, in addition to local pulmonary effects, which may explain the different effects of mitoTEMPO/TPP on edema formation in vivo and in the isolated lung. Inhibition of ROS generation in macrophages may mitigate epithelium damage and prevent edema formation in vivo but not ex vivo. Moreover, time-dependent effects may play a role, as in the isolated lung, edema formation was determined $150 \mathrm{~min}$ after LPS application (in vivo after $24 \mathrm{~h}$ ). MitoTEMPO was applied in the isolated lung in a therapeutic manner $120 \mathrm{~min}$ after LPS application when edema formation was already triggered. This suggests that both ROS originating from circulating immune cells and ROS formed in lung tissue contribute to the induction of edema. In contrast, an increase in PAP is a result of interaction between endothelial cells and LPS. Thus, we assume that there are two separate mechanisms mediated by ROS, causing either increased PAP or the development of edema. This assumption adequately explains the action of reduced ROS on PAP and edema. Further studies are required to understand the interaction between mitochondria-targeted antioxidants and lung tissue in detail.

Conclusively, our findings suggest a different role of lung mitochondria for the development of the pathological effects associated with systemic inflammation, compared to the other organs. As TPP is sufficient for mitigating those effects, it may become a useful therapeutic intervention that specifically targets the lung.

Supplementary Materials: The following supporting information can be downloaded at: https: / /www.mdpi.com/article/10.3390/antiox11020323/s1, Supplementary Materials S1: Preparation of lung tissue slices, Figure S1: LSM images of precision cut lung slices.

Author Contributions: Conceptualization, A.V.K.; methodology, A.W., J.C.D., N.S., M.H. and A.V.K.; validation, all coauthors.; formal analysis L.B., A.T.M., S.Z., A.M., J.S. and M.H.; writing—original draft preparation, A.W.; writing—-review and editing, all coauthors; visualization, A.W., J.C.D., N.S., A.W., J.C.D. and N.S.; funding acquisition, N.S. and A.V.K. All authors have read and agreed to the published version of the manuscript.

Funding: This work was supported by Österreichische Forschungsförderungsgesellschaft mbH (FFG) Projekt number FO999887791 and by Deutsche Forschungsgemeinschaft (DFG, German Research Foundation)—Project number 268555672—SFB 1213, Project A06.

Institutional Review Board Statement: All interventions were conducted in compliance with the Guide for the Care and Use of Laboratory Animals published by the National Institute of Health with approval from the Animal Protocol Review Board (Austria no. 815758/2014/16; Germany no. GI 105/2014).

Informed Consent Statement: Not applicable.

Data Availability Statement: Data are contained within the article and supplementary material.

Acknowledgments: The authors thank Andreas Gasser for technical support and James Ferguson for linguistic proofread of this manuscript.

Conflicts of Interest: The authors declare no conflict of interest. 


\section{Appendix A}

Table A1. Sequences for primers used for measuring gene expression. HPRT: hypoxanthine-guanine phosphoribosyl transferase; CHOP: CCAAT/enhancer-binding protein homologous protein; IL6: interleukin 6; iNOS: inducible nitric oxide synthase; HO1: heme oxygenase 1; NFkBia: NFkB inhibitor alpha; TNFR1: tumor necrosis factor receptor 1.

\begin{tabular}{|c|c|c|c|}
\hline Gene & $\begin{array}{l}\text { Accession } \\
\text { Number }\end{array}$ & Primer Sequence & Source \\
\hline Cyclophilin A & M19533 & $\begin{array}{l}\text { F: TAT CTG CAC TGC CAA GAC TGA } \\
\text { GTG } \\
\text { R: CTT CTT GCT GGT CTT GCC ATT CC }\end{array}$ & [24] \\
\hline HPRT & NM_012583 & $\begin{array}{l}\text { F: CTC ATG GAC TGA TTA TGG ACA } \\
\text { GGA C } \\
\text { R: GCA GGT CAG CAA AGA ACT TAT } \\
\text { AGC C }\end{array}$ & [24] \\
\hline $\mathrm{CHOP}$ & NM_024134.2 & $\begin{array}{l}\text { F: TTG GGG GCA CCT ATA TCT CA } \\
\text { R: CTC CTT CAG TCG CTG TTT CC }\end{array}$ & [24] \\
\hline IL6 & NM_012589.1 & $\begin{array}{l}\text { F: CCG GAG AGG AGA CTT CAC AG } \\
\text { R: ACA GTG CAT CAT CGC TGT TC }\end{array}$ & [23] \\
\hline iNOS & NM_012611.3 & $\begin{array}{l}\text { F: AGG CAA GCC CTC ACC TAC TT } \\
\text { R: GTG GGG TTG TTG CTG AAC TT }\end{array}$ & [23] \\
\hline HO1 & NM_012580.2 & $\begin{array}{l}\text { F: CCA GCC ACA CAG CAC TAC } \\
\text { R: GCG GTC TTA GCC TCT TCT G }\end{array}$ & [24] \\
\hline NFkBia & NM_001105720.2 & $\begin{array}{l}\text { F: GCA ACA GCT CAC GGA GGA CGG } \\
\text { R: TCC ACG ATG CCC AGG TAG CCA }\end{array}$ & $\begin{array}{l}\text { Desinged for } \\
\text { this study }\end{array}$ \\
\hline TNFR1 & NM_013091.1 & $\begin{array}{c}\text { F: GTG CCA CAA AGG AAC CTA CTT G } \\
\text { R: AGG CTG GAG TTA GGG GCT TA }\end{array}$ & [38] \\
\hline
\end{tabular}

\section{References}

1. Skulachev, V.P. How to clean the dirtiest place in the cell: Cationic antioxidants as intramitochondrial ROS scavengers. IUBMB Life 2005, 57, 305-310. [CrossRef]

2. Patil, N.K.; Parajuli, N.; MacMillan-Crow, L.A.; Mayeux, P.R. Inactivation of renal mitochondrial respiratory complexes and manganese superoxide dismutase during sepsis: Mitochondria-targeted antioxidant mitigates injury. Am. J. Physiol. Renal Physiol. 2014, 306, F734-F743. [CrossRef]

3. Li, S.; Wu, H.; Han, D.; Ma, S.; Fan, W.; Wang, Y.; Zhang, R.; Fan, M.; Huang, Y.; Fu, X.; et al. A Novel Mechanism of Mesenchymal Stromal Cell-Mediated Protection against Sepsis: Restricting Inflammasome Activation in Macrophages by Increasing Mitophagy and Decreasing Mitochondrial ROS. Oxid. Med. Cell. Longev. 2018, 2018, 3537609. [CrossRef] [PubMed]

4. Arulkumaran, N.; Pollen, S.J.; Tidswell, R.; Gaupp, C.; Peters, V.B.M.; Stanzani, G.; Snow, T.A.C.; Duchen, M.R.; Singer, M. Selective mitochondrial antioxidant MitoTEMPO reduces renal dysfunction and systemic inflammation in experimental sepsis in rats. Br. J. Anaesth. 2021, 127, 577-586. [CrossRef] [PubMed]

5. Song, Y.; Xing, H.; He, Y.; Zhang, Z.; Shi, G.; Wu, S.; Liu, Y.; Harrington, E.O.; Sellke, F.W.; Feng, J. Inhibition of mitochondrial reactive oxygen species improves coronary endothelial function after cardioplegic hypoxia/reoxygenation. J. Thorac. Cardiovasc. Surg. 2021. [CrossRef] [PubMed]

6. Weidinger, A.; Müllebner, A.; Paier-Pourani, J.; Banerjee, A.; Miller, I.; Lauterböck, L.; Duvigneau, J.C.; Skulachev, V.P.; Redl, H.; Kozlov, A.V. Vicious Inducible Nitric Oxide Synthase-Mitochondrial Reactive Oxygen Species Cycle Accelerates Inflammatory Response and Causes Liver Injury in Rats. Antioxid. Redox Signal. 2015, 22, 572-586. [CrossRef]

7. Rademann, P.; Weidinger, A.; Drechsler, S.; Meszaros, A.; Zipperle, J.; Jafarmadar, M.; Dumitrescu, S.; Hacobian, A.; Ungelenk, L.; Röstel, F.; et al. Mitochondria-targeted antioxidants SkQ1 and MitoTEMPO failed to exert a long-term beneficial effect in murine polymicrobial sepsis. Oxid. Med. Cell. Longev. 2017, 2017, 6412682. [CrossRef]

8. Powell, R.D.; Goodenow, D.A.; Britton Christmas, A.; McKillop, I.H.; Evans, S.L. Effect of systemic triphenylphosphonium on organ function and oxidative stress. Am. Surg. 2018, 84, 36-42. [CrossRef]

9. Ozsvari, B.; Sotgia, F.; Lisanti, M.P. Exploiting mitochondrial targeting signal(s), TPP and bis-TPP, for eradicating cancer stem cells (CSCs). Aging 2018, 10, 229-240. [CrossRef]

10. Trnka, J.; Elkalaf, M.; Andě, M. Lipophilic triphenylphosphonium cations inhibit mitochondrial electron transport chain and induce mitochondrial proton leak. PLoS ONE 2015, 10, e0121837. [CrossRef] 
11. Cadenas, S. Mitochondrial uncoupling, ROS generation and cardioprotection. Biochim. Biophys. Acta-Bioenerg. 2018, 1859, 940-950. [CrossRef] [PubMed]

12. Weidinger, A.; Kozlov, A.V. Biological Activities of Reactive Oxygen and Nitrogen Species: Oxidative Stress versus Signal Transduction. Biomolecules 2015, 5, 472-484. [CrossRef] [PubMed]

13. Speer, C.P. New insights into the pathogenesis of pulmonary inflammation in preterm infants. Biol. Neonate 2001, 79, 205-209. [PubMed]

14. Brooks, D.; Barr, L.C.; Wiscombe, S.; McAuley, D.F.; Simpson, A.J.; Rostron, A.J. Human lipopolysaccharide models provide mechanistic and therapeutic insights into systemic and pulmonary inflammation. Eur. Respir. J. 2020, 56, 1901298. [CrossRef] [PubMed]

15. Zuo, L.; Otenbaker, N.P.; Rose, B.A.; Salisbury, K.S. Molecular mechanisms of reactive oxygen species-related pulmonary inflammation and asthma. Mol. Immunol. 2013, 56, 57-63. [CrossRef] [PubMed]

16. Kratzer, E.; Tian, Y.; Sarich, N.; Wu, T.; Meliton, A.; Leff, A.; Birukova, A.A. Oxidative stress contributes to lung injury and barrier dysfunction via microtubule destabilization. Am. J. Respir. Cell Mol. Biol. 2012, 47, 688-697. [CrossRef]

17. Gandhirajan, R.K.; Meng, S.; Chandramoorthy, H.C.; Mallilankaraman, K.; Mancarella, S.; Gao, H.; Razmpour, R.; Yang, X.-F.; Houser, S.R.; Chen, J.; et al. Blockade of NOX2 and STIM1 signaling limits lipopolysaccharide-induced vascular inflammation. J. Clin. Investig. 2013, 123, 887-902. [CrossRef]

18. Fu, P.; Epshtein, Y.; Ramchandran, R.; Mascarenhas, J.B.; Cress, A.E.; Jacobson, J.; Garcia, J.G.N.; Natarajan, V. Essential role for paxillin tyrosine phosphorylation in LPS-induced mitochondrial fission, ROS generation and lung endothelial barrier loss. Sci. Rep. 2021, 11, 17546. [CrossRef] [PubMed]

19. Song, J.-A.; Yang, H.-S.; Lee, J.; Kwon, S.; Jung, K.J.; Heo, J.-D.; Cho, K.-H.; Song, C.W.; Lee, K. Standardization of bronchoalveolar lavage method based on suction frequency number and lavage fraction number using rats. Toxicol. Res. 2010, 26, 203-208. [CrossRef]

20. Ray, A.; Dittel, B.N. Isolation of mouse peritoneal cavity cells. J. Vis. Exp. 2010, 35, e1488. [CrossRef]

21. Zhou, M.; Diwu, Z.; Panchuk-Voloshina, N.; Haugland, R.P. A stable nonfluorescent derivative of resorufin for the fluorometric determination of trace hydrogen peroxide: Applications in detecting the activity of phagocyte NADPH oxidase and other oxidases. Anal. Biochem. 1997, 253, 162-168. [CrossRef] [PubMed]

22. Jais, A.; Einwallner, E.; Sharif, O.; Gossens, K.; Lu, T.T.-H.; Soyal, S.M.; Medgyesi, D.; Neureiter, D.; Paier-Pourani, J.; Dalgaard, K.; et al. Heme oxygenase-1 drives metaflammation and insulin resistance in mouse and man. Cell 2014, 158, 25-40. [CrossRef] [PubMed]

23. Weidinger, A.; Dungel, P.; Perlinger, M.; Singer, K.; Ghebes, C.; Duvigneau, J.C.; Müllebner, A.; Schäfer, U.; Redl, H.; Kozlov, A.V. Experimental data suggesting that inflammation mediated rat liver mitochondrial dysfunction results from secondary hypoxia rather than from direct effects of inflammatory mediators. Front. Physiol. 2013, 4, 138. [CrossRef]

24. Müllebner, A.; Moldzio, R.; Redl, H.; Kozlov, A.V.; Duvigneau, J.C. Heme Degradation by Heme Oxygenase Protects Mitochondria but Induces ER Stress via Formed Bilirubin. Biomolecules 2015, 5, 679-701. [CrossRef] [PubMed]

25. Sommer, N.; Hüttemann, M.; Pak, O.; Scheibe, S.; Knoepp, F.; Sinkler, C.; Malczyk, M.; Gierhardt, M.; Esfandiary, A.; Kraut, S.; et al. Mitochondrial Complex IV Subunit 4 Isoform 2 Is Essential for Acute Pulmonary Oxygen Sensing. Circ. Res. 2017, 121, 424-438. [CrossRef]

26. Park, W.H. Tempol differently affects cellular redox changes and antioxidant enzymes in various lung-related cells. Sci. Rep. 2021, 11, 14869. [CrossRef]

27. Jirillo, E.; Caccavo, D.; Magrone, T.; Piccigallo, E.; Amati, L.; Lembo, A.; Kalis, C.; Gumenscheimer, M. The role of the liver in the response to LPS: Experimental and clinical findings. J. Endotoxin Res. 2002, 8, 319-327. [CrossRef]

28. Wideman, R.F.; Bowen, O.T.; Erf, G.F. Broiler pulmonary hypertensive responses during lipopolysaccharide-induced tolerance and cyclooxygenase inhibition. Poult. Sci. 2009, 88, 72-85. [CrossRef]

29. Sheng, S.-J.; Nie, Y.-C.; Lin, F.; Li, P.-B.; Liu, M.-H.; Xie, C.-S.; Long, C.-F.; Su, W.-W. Biphasic modulation of $\alpha$-ENaC expression by lipopolysaccharide in vitro and in vivo. Mol. Med. Rep. 2014, 10, 773-777. [CrossRef]

30. Dauphinee, S.M.; Karsan, A. Lipopolysaccharide signaling in endothelial cells. Lab. Investig. 2006, 86, 9-22. [CrossRef]

31. López-Ongil, S.; Senchak, V.; Saura, M.; Zaragoza, C.; Ames, M.; Ballermann, B.; Rodríguez-Puyol, M.; Rodríguez-Puyol, D.; Lowenstein, C.J. Superoxide regulation of endothelin-converting enzyme. J. Biol. Chem. 2000, 275, 26423-26427. [CrossRef] [PubMed]

32. Pak, O.; Scheibe, S.; Esfandiary, A.; Gierhardt, M.; Sydykov, A.; Logan, A. Impact of the mitochondria-targeted antioxidant MitoQ on hypoxia-induced pulmonary hypertension. Eur. Respir. J. 2018, 51, 1701024. [CrossRef]

33. Wynne, B.M.; Zou, L.; Linck, V.; Hoover, R.S.; Ma, H.-P.; Eaton, D.C. Regulation of Lung Epithelial Sodium Channels by Cytokines and Chemokines. Front. Immunol. 2017, 8, 766. [CrossRef]

34. Mittal, M.; Siddiqui, M.R.; Tran, K.; Reddy, S.P.; Malik, A.B. Reactive oxygen species in inflammation and tissue injury. Antioxid. Redox Signal. 2014, 20, 1126-1167. [CrossRef] [PubMed]

35. Burghuber, O.; Mathias, M.M.; McMurtry, I.F.; Reeves, J.T.; Voelkel, N.F. Lung edema due to hydrogen peroxide is independent of cyclooxygenase products. J. Appl. Physiol. 1984, 56, 900-905. [CrossRef]

36. Tzeng, H.-P.; Ho, F.-M.; Chao, K.-F.; Kuo, M.-L.; Lin-Shiau, S.-Y.; Liu, S.-H. beta-Lapachone reduces endotoxin-induced macrophage activation and lung edema and mortality. Am. J. Respir. Crit. Care Med. 2003, 168, 85-91. [CrossRef] [PubMed] 
37. Nakamura, M.; Motoyama, S.; Saito, S.; Minamiya, Y.; Saito, R.; Ogawa, J. Hydrogen peroxide derived from intestine through the mesenteric lymph induces lung edema after surgical stress. Shock 2004, 21, 160-164. [CrossRef]

38. Mkrtchyan, G.V.; Üçal, M.; Müllebner, A.; Dumitrescu, S.; Kames, M.; Moldzio, R.; Molcanyi, M.; Schaefer, S.; Weidinger, A.; Schaefer, U.; et al. Thiamine preserves mitochondrial function in a rat model of traumatic brain injury, preventing inactivation of the 2-oxoglutarate dehydrogenase complex. Biochim. Biophys. Acta. Bioenerg. 2018, 1859, 925-931. [CrossRef] 